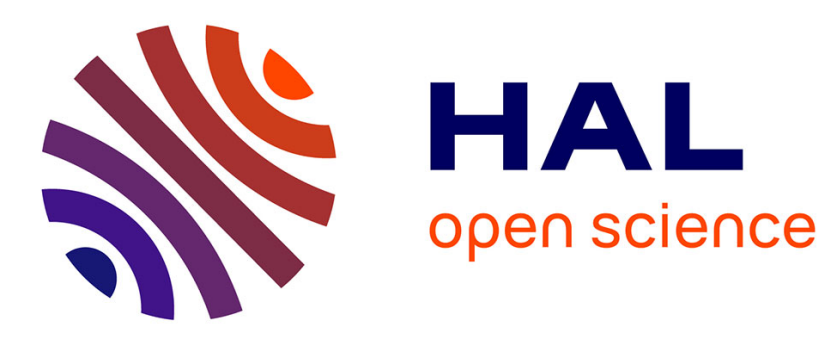

\title{
Introduction of an internal time in nonlocal integral theories
}

\author{
Rodrigue Desmorat, Fabrice Gatuingt
}

\section{To cite this version:}

Rodrigue Desmorat, Fabrice Gatuingt. Introduction of an internal time in nonlocal integral theories. 2007. hal-00200898

\section{HAL Id: hal-00200898 \\ https://hal.science/hal-00200898}

Submitted on 22 Dec 2007

HAL is a multi-disciplinary open access archive for the deposit and dissemination of scientific research documents, whether they are published or not. The documents may come from teaching and research institutions in France or abroad, or from public or private research centers.
L'archive ouverte pluridisciplinaire HAL, est destinée au dépôt et à la diffusion de documents scientifiques de niveau recherche, publiés ou non, émanant des établissements d'enseignement et de recherche français ou étrangers, des laboratoires publics ou privés. 


\title{
Introduction of an internal time in nonlocal integral theories
}

\author{
Internal report $\mathrm{LMT} \mathrm{n}^{\circ} 268$ \\ ISBN : 978-2-11-097305-4
}

\author{
R. Desmorat and F. Gatuingt \\ LMT-Cachan \\ ENS Cachan/CNRS/Université Paris 6/PRES Universud Paris \\ 61, av. du Président Wilson \\ F-94230 Cachan, France \\ 2007
}




\begin{abstract}
Nonlocal damage models are now commonly used. Their ability to make finite element computations with softening laws robust and mesh independent is well established. There are nevertheless still a few open questions as the identification of the so-called internal length $l_{c}$, as its loading or its boundray independency. One focus in the present note on the boundary conditions problem and on the feature that points separated by a crack or a hole should not interact as they do in Pijaudier-Cabot and Bazant initial nonlocal theory. Instead of defining an internal length one proposes to make the nonlocal weight function as a function of the information time propagation of an elastic wave normalized by an internal time $\tau_{c}$.
\end{abstract}

Key-words : nonlocal; damage; negative hardening

\title{
Résumé
}

Les modèles d'endommagement nonlocaux sont désormais robustes et couramment utilisés dans le cadre de calculs numériques sur structures complexes. La question des conditions aux limites demeure néanmoins non résolue, de même que l'identification et la question de la dépendance structurelle de la longueur interne habituellement introduite $l_{c}$. Nous nous attachons dans la présente note à proposer une théorie nonlocale de type intégral évitant l'interaction des points de part et d'autre des lèvres d'une fissure, d'entailles ou de trous, théorie basée sur le temps de propagation d'ondes élastiques. Un temps interne est considéré en lieu et place de la longueur interne.

Mots-clés : nonlocal; endommagement; écrouissage négatif 


\section{Contents}

1 Wave propagation $\quad 7$

1.1 Finite Element modelling . . . . . . . . . . . . . . . . . . . 7

1.2 Propagation in plain media . . . . . . . . . . . . . . . . 8

1.3 Propagation in notched media . . . . . . . . . . . . . . . . . . . 9

1.4 Propagation in damaged media . . . . . . . . . . . . . . . . . . . . . . . . . . . . . . . . . . . 10

1.5 Discussion . . . . . . . . . . . . . . . . . . . . . 11

2 Nonlocal theory with internal time $\quad 13$

2.1 Nonlocal integral theories - Boundary effect . . . . . . . . . . . . . . 13

2.2 Time instead of distance . . . . . . . . . . . . . . . . . . . . . 14

2.3 Example: nonlocal damage models . . . . . . . . . . . . . . . . . . 15

2.4 Effective or "dynamic" distance - Vanishing internal length . . . . . . . . . 16

3 Equivalence crack / damaged zone 19

3.1 Connectivity matrices . . . . . . . . . . . . . . . . . . . . 20

3.2 Straight 1D wave propagation . . . . . . . . . . . . . . . 22

4 Bar with a damaged zone $\quad 27$ 


\section{Introduction}

The effect and the formulation of boundary conditions - such as free edges, notches and initial cracks - remain an open question for nonlocal models. The main drawback of the classical nonlocal integral theory [1] consists in the nonphysical interaction, through the nonlocal averaging process, of points across a crack or a hole. The definition of natural boundary conditions of vanishing strain normal derivative at a free edge is still under discussion for gradient formulations [2,3]. The continuous nucleation of a crack of zero thickness is not so simple as the thickness of a localization band is more or less proportional to the internal length introduced. Local behavior along free edges - i.e. with a vanishing internal length - has been obtained by some authors [4]. The consideration of an internal length evolving with damage $[5,6]$ seems a way to properly bridge Damage Mechanics and Fracture Mechanics as the internal length may then vanish for large values of damage.

In the present note, one proposes a solution - bringing also questions - to these main difficulties. The idea is to keep the nonlocal averaging process but to quantify the distance between points as an effective distance, i.e. as a distance function for instance of the geometry and the matter encountered beween interacting points. One proposes to define such an effective distance with respect to a dynamic process: with respect to how information or wave propagates between interacting points. This can be made through the introduction of an internal time, constant, instead of a internal length, measured as evolving. Dynamics is important to define a link between a characteristic time and a characteristic length, either when viscosity is introduced [7,9] or when the physical defects

obscuration phenomenon encountered in high speed dynamics and multi-fragmentation is taken into account [8]. Some authors even introduce the classical nonlocal theory by comparing the characteristic wavelength of the deformation field to an intrinsic length of the material [10], still a dynamics vocabulary. Remind also that the physics is often dynamic at small (discrete) scales as in quantuum mechanics, as in dislocations theories or as at the scale of the macromolecular chains in elastomers, even if the continuum macroscopic behavior is quasi-static. Let us then accept then the importance of the dynamics effects and of wave propagations. But as just mentioned in order to address quasi-static non-viscous structural failure. 


\section{Chapter 1}

\section{Wave propagation in plain, notched and damaged media}

This part illustrates the wave propagation in different media. It contributes to the understanding of the differences or similarities between a notched and a damaged zone.

\subsection{Finite Element modelling}

In this chapter one considers the problem of an impact on a square plate of the Figure 1.1 $(1.5 \mathrm{~m} \times 1.5 \mathrm{~m} \times 0.01 \mathrm{~m})$. An impulse force $F(t)$ according to the out of plane $z$-axis (close to a Dirac) is applied to the plate (Figure 1.2) in order to generate a wave propagation in this plate.

In order to numerically simulate this problem, one uses the Finite Element code Dyna3D to obtain the transient solution of a wave propagation. The plate is modeled with $3 \mathrm{D}$ under-integrated brick elements with an elastic material ( $\mathrm{E}=35 \mathrm{GPa}, \nu=0,2, \rho=2400$ $\mathrm{kg} / \mathrm{m}^{3}$ ). No boundaries conditions are applied in the simulations. The discrete equations of motion are solved with an explicit scheme so that the time step is directly linked to the element size (Courant's condition satisfied). In order to gain a good description of the wave propagation, one choses to have small elements $(0.015 \mathrm{~m} \times 0.015 \mathrm{~m} \times 0.01 \mathrm{~m})$. One obtains then approximately 360000 elements with a time step equal to $5 \cdot 10^{-4} \mathrm{~ms}$. This time step will be the accuracy of the different computed times in the problem. 


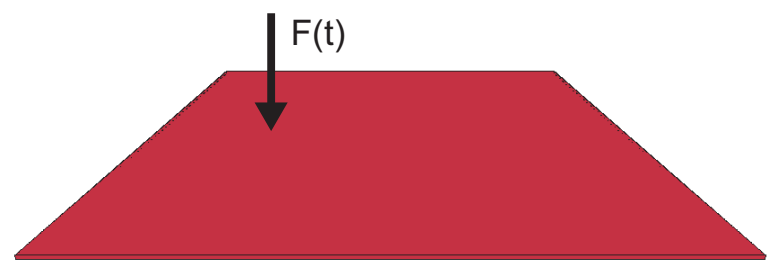

Figure 1.1: Impact on a square plate.

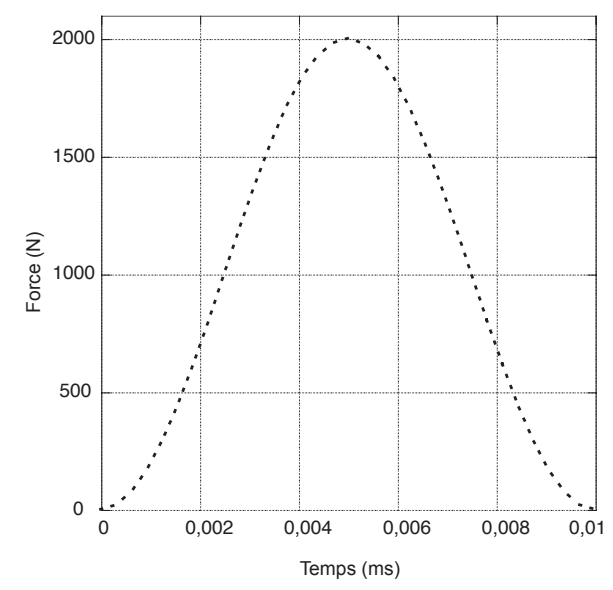

Figure 1.2: Force vs time for the impact force.

\subsection{Propagation in plain media}

Figure 1.3 shows the specimen used to model the wave propagation in the plain plate. Figure 1.4 shows the map of the $z$-acceleration in the plate at a time $t=100 \mathrm{~ms}$. Notice that one can see the wave reflection on the free faces of the plate (up and left). 


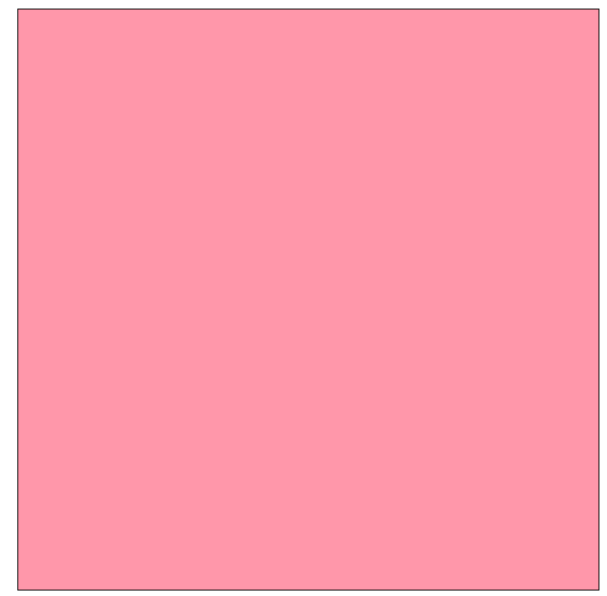

Figure 1.3: Plain specimen.

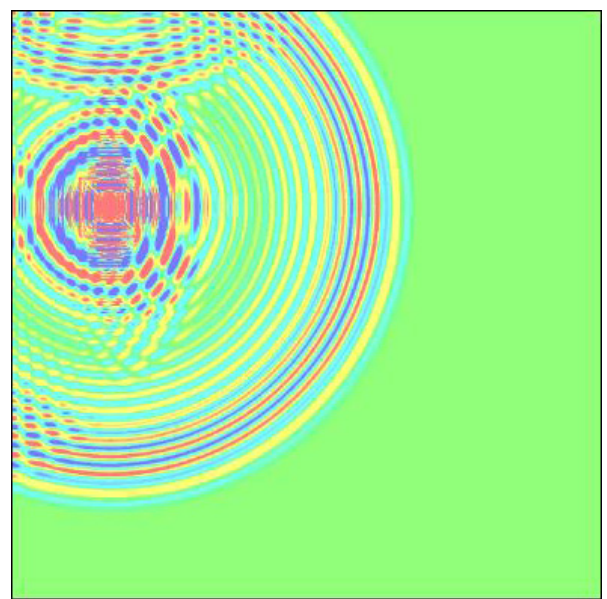

Figure 1.4: Wave propagation in the plain specimen.

\subsection{Propagation in notched media}

Figure 1.5 shows the specimen used to model the wave propagation in the notched plate. The length of the notch is $0.5 \mathrm{~m}$, its width $0.1 \mathrm{~m}$. Figure 1.6 shows the map of the $z$-acceleration in the plate at $\mathrm{t}=100 \mathrm{~ms}$. Notice again that one can see the wave reflection on the free faces of the plate and in particular the reflection due to the notch (upper lip). 


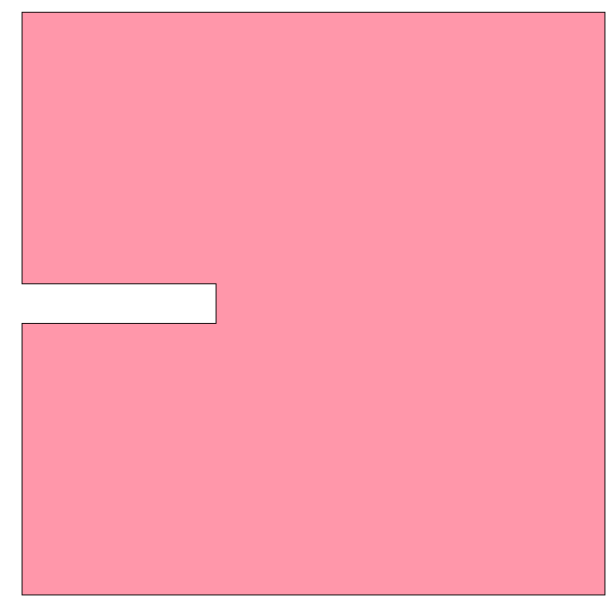

Figure 1.5: Notched specimen.

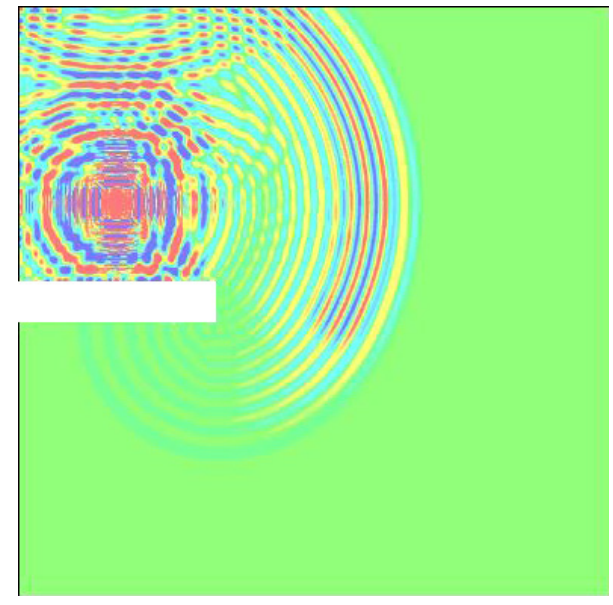

Figure 1.6: Wave propagation in the notched specimen.

\subsection{Propagation in damaged media}

Figure 1.7 shows the specimen used to model the wave propagation in a plate where previous notch is replaced by a damaged material at a damage level $\mathrm{D}=0.99$. In the computation one uses for the damaged zone a constant effective Young's modulus $\tilde{E}=$ $E(1-D)$ instead of the initial modulus $E$. This means here $\tilde{E}=E / 100$. Figure 1.8 shows the map of the $z$-acceleration in the plate at $\mathrm{t}=100 \mathrm{~ms}$. One sees the wave reflection on the free edges of the plate as in the previous cases. It is important to see that in this case, the damaged zone acts almost as a free surface for the wave propagation. This is due to the large differences in impedance between the initial undamaged material and the 
damaged one.

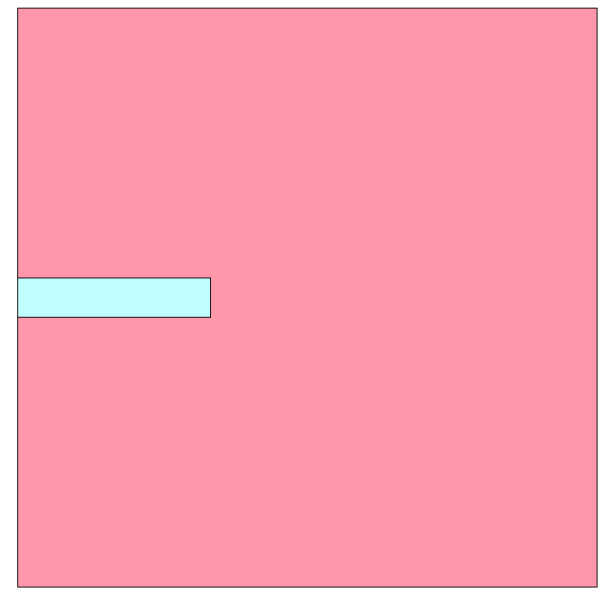

Figure 1.7: Damaged specimen.

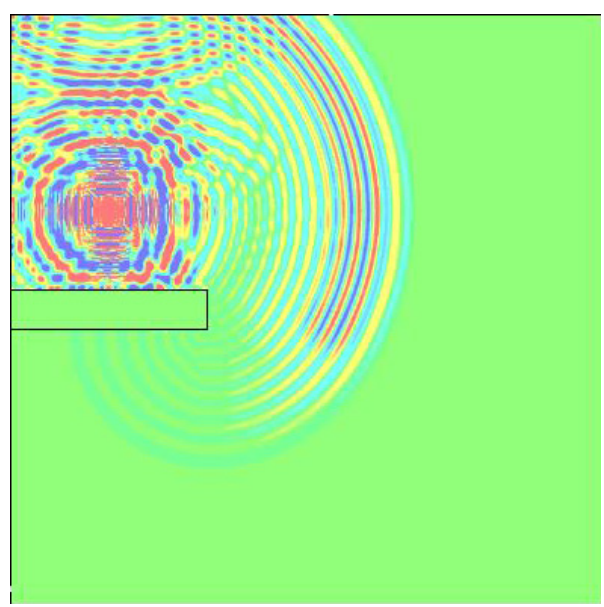

Figure 1.8: Wave propagation in the "damaged" specimen.

\subsection{Discussion}

These simple examples illustrate the wave propagation without and with notches (nothing new). First, wave propagation is affected by the free edges (classical reflection). Second, the comparison between the real notch and the damaged zone allows to conclude that highly damaged material (low Young's modulus) behaves as a notch. 
These two properties are the basis of the nonlocal integral formulation proposed next. 


\section{Chapter 2}

\section{Nonlocal theory with internal time}

Softening constitutive equations classically lead to spurious dissipation modes and to mesh dependency. The need of the definition - and the introduction - of an internal length in the models is now established. But in which form ? In a gradient form ? In an inegral form ? From an internal viscosity (delay-damage) combined then with dynamics ? The main idea of such regularizations is to average the "engine" - i.e. the thermodynamics force, denoted next $\mathcal{V}$ in the general case or $Y$ or $\hat{\epsilon}$ for damage - responsible for the strain localization. The procedure to define a nonlocal variable $\mathcal{V}^{\text {nl }}$ from its local expression $\mathcal{V}$ introduces a characteristic length $l_{c}$ considered as a material parameter.

One do not intend here to solve the problem in the general case and focus only on the nonlocal integral theories.

\subsection{Nonlocal integral theories - Boundary effect}

The classical nonlocal theory [1] uses the integral

$$
\mathcal{V}^{n l}(\boldsymbol{x})=\frac{1}{V_{r}} \int_{\Omega} \psi\left(\frac{\|\boldsymbol{x}-\boldsymbol{s}\|}{l_{c}}\right) \mathcal{V}(\boldsymbol{s}) d s \quad V_{r}(\boldsymbol{x})=\int_{\Omega} \psi\left(\frac{\|\boldsymbol{x}-\boldsymbol{s}\|}{l_{c}}\right) d s
$$

over the whole domain $\Omega$ in order to define a nonlocal quantity $\mathcal{V}^{n l}$ - built from the local variable $\mathcal{V}$ - to be used in thermodynamics state or evolution laws.

In Eq. (2.1) $\psi$ is the nonlocal weight function, positive and decreasing with respect to the distance $\|x-s\|$ between the considered point $\boldsymbol{x}$ and all the structure points $\boldsymbol{s}$. The normalizing factor is denoted $V_{r}(\boldsymbol{x})$ and $l_{c}$ is an internal or characteristic length. Two classical $\psi$-functions are

$$
\psi(\xi)=e^{-\frac{1}{2} \xi^{2}} \quad \text { or } \quad \psi(\xi)=\left\langle 1-\xi^{2}\right\rangle^{2}
$$

The expressions (2.1) do then define the same contribution to $\mathcal{V}^{n l}$ of points $\boldsymbol{x}$ and $\boldsymbol{s}$ 
- across a crack than any points $\boldsymbol{x}$ and $\boldsymbol{s}$ separated by the same distance $\|\boldsymbol{x}-\boldsymbol{s}\|$ (for instance path $\boldsymbol{x}_{1}-\boldsymbol{x}_{6}$ versus path $\boldsymbol{x}_{2}-\boldsymbol{x}_{5}$ of figure 2.1 ),

- across a damaged zone: for example the interaction between points $x_{3}-x_{4}$ is not affected by the zone at a damage level $D_{0}$, i.e. at a Young's $E\left(1-D_{0}\right)$ much lower than the Young's modulus of the virgin material.

This illustrates the need to deal with such a boudary condition effect but also to make equivalent a real crack and a highly damaged zone.

Specimen (a)

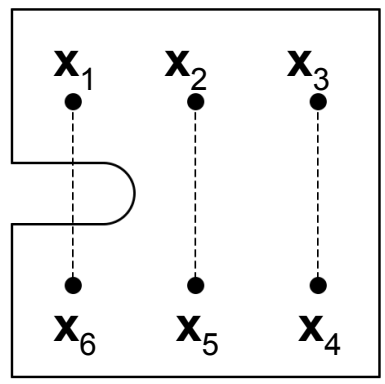

Specimen (b)

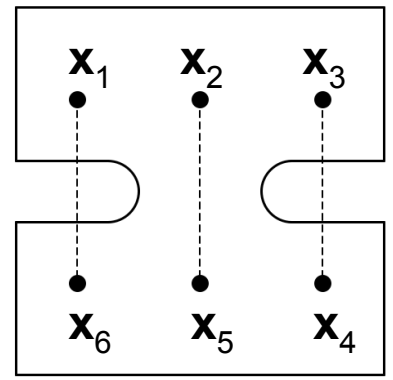

Specimen (c)

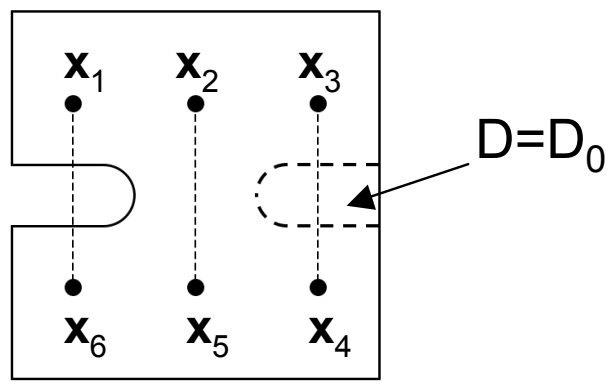

Figure 2.1: Notched and damaged specimens.

\subsection{Time instead of distance}

In order to solve the problem, one proposes in this work to keep the nonlocal integral framework and to consider the information time propagation $\tau_{x s}$ between points $\boldsymbol{x}$ and $\boldsymbol{s}$ instead of the classical distance $\|\boldsymbol{x}-\boldsymbol{s}\|$.

A nonlocal theory with internal time is then simply defined by replacing Eq. (2.1) by:

$$
\mathcal{V}^{n l}(\boldsymbol{x})=\frac{1}{V_{r}} \int_{\Omega} \psi\left(\frac{\tau_{x s}}{\tau_{c}}\right) \mathcal{V}(\boldsymbol{s}) d s \quad V_{r}(\boldsymbol{x})=\int_{\Omega} \psi\left(\frac{\tau_{x s}}{\tau_{c}}\right) d s
$$

with $\tau_{x s}$ the information propagation time taken next as the time for a wave to propagate from point $\boldsymbol{x}$ to point $\boldsymbol{s}$ and $\tau_{c}$ a material parameter. As the wave time propagation $\tau_{s x}$ from point $\boldsymbol{s}$ to point $\boldsymbol{x}$ is identical to $\tau_{x s}$ the nonlocal weight function thus built is symmetric.

Such a nonlocal averaging process applies to physical laws of different nature.

Concerning continuum mechanics and elasticity, plasticity and/or damage, the variables $\mathcal{V}$ are often equivalent strains, accumulated plastic strains or strain energy densities. 


\subsection{Example: nonlocal damage models}

For example, local isotropic damage theories for quasi-brittle materials define

- the damage as a loss of stiffness $D=1-\tilde{E} / E$ with $\tilde{E}$ (resp. $E$ ) the damaged (resp. initial) Young modulus, equation also rewritten in 3D as the elasticity law coupled with isotropic damage

$$
\sigma=\underline{E}(1-D): \epsilon
$$

with $\boldsymbol{\sigma}, \boldsymbol{\epsilon}$ and $\underline{\boldsymbol{E}}$ respectively the stress, the strain and the Hooke tensors.

- the damage evolution as a function of a local variable $\mathcal{V}$ either equal to the thermodynamics force $Y=\frac{1}{2} \boldsymbol{\epsilon}: \underline{\boldsymbol{E}}: \boldsymbol{\epsilon}$ associated with damage (Marigo model) or to an equivalent strain as Mazars strain $\hat{\epsilon}=\sqrt{\langle\boldsymbol{\epsilon}\rangle_{+}:\langle\boldsymbol{\epsilon}\rangle_{+}}$,

$$
D=g(\mathcal{V})
$$

with $g$ a nonlinear function.

The non local damage law is simply written

$$
D=g\left(\mathcal{V}^{n l}\right)
$$

instead of Eq. (2.5) with the nonlocal averaging process (2.3).

For concrete, the microcracks due to tension are mainly orthogonal to the loading direction, when the microcracks due to compression are mainly parallel to the loading direction. The damage state has then to be represented by a tensorial variable $\boldsymbol{D}$ either a fourth rank tensor or a second rank tensor. The use of a second order damage tensor is more convenient for practical applications (as well as for the material parameters identification) and this is the choice which has been made. The damage anisotropy induced by either tension or compression is simply modeled by the consideration of damage evolution laws ensuring a damage rate proportional to the positive part of the strain tensor, i.e. a damage governed by the principal extensions [11].

The full set of constitutive equations for the local anisotropic damage model reads $[12,13]$

- Elasticity,

$$
\boldsymbol{\epsilon}=\frac{1+\nu}{E} \tilde{\boldsymbol{\sigma}}-\frac{\nu}{E} \operatorname{tr} \tilde{\boldsymbol{\sigma}} 1
$$

with $E$ the Young modulus and $\nu$ the Poisson ratio. 
- Effective stress,

$$
\tilde{\boldsymbol{\sigma}}=\left((\mathbf{1}-\boldsymbol{D})^{-1 / 2} \boldsymbol{\sigma}^{D}(\mathbf{1}-\boldsymbol{D})^{-1 / 2}\right)^{D}+\frac{1}{3}\left[\frac{\langle\operatorname{tr} \boldsymbol{\sigma}\rangle}{1-\operatorname{tr} \boldsymbol{D}}-\langle-\operatorname{tr} \boldsymbol{\sigma}\rangle\right] \mathbf{1}
$$

where(. $)^{D}$ stands for the deviatoric part and $\langle$.$\rangle for the positive part of a scalar.$

- Damage criterion,

$$
f=\hat{\epsilon}-\kappa(\operatorname{tr} \boldsymbol{D})
$$

so that the condition $f<0 \rightarrow$ elastic loading or unloading, $f=0, \dot{f}=0 \rightarrow$ damage growth, where $\hat{\epsilon}=\sqrt{\langle\boldsymbol{\epsilon}\rangle_{+}:\langle\boldsymbol{\epsilon}\rangle_{+}}$is Mazars equivalent strain built from the positive part $\langle\boldsymbol{\epsilon}\rangle_{+}$of the strain tensor and where

$$
\kappa(\operatorname{tr} \boldsymbol{D})=a \cdot \tan \left[\frac{\operatorname{tr} \boldsymbol{D}}{a A}+\arctan \left(\frac{\kappa_{0}}{a}\right)\right]
$$

- Induced damage anisotropy governed by the positive extensions,

$$
D=\dot{\lambda}\langle\boldsymbol{\epsilon}\rangle_{+}^{2}
$$

In the rate independent formulation, the damage multiplier $\dot{\lambda}$ is determined from the consistency condition $f=0, \dot{f}=0$.

There are 5 material parameters introduced: $E, \nu$ for elasticity, $\kappa_{0}$ as damage threshold and $A$ and $a$ as damage parameters.

The model is simply made nonlocal, either from the classical integral theory or from the new integral nonlocal with internal time theory, by replacing Mazars equivalent strain $\hat{\epsilon}$ by its nonlocal form in the damage criterion function, becoming

$$
f=\hat{\epsilon}^{n l}-\kappa(\operatorname{tr} \boldsymbol{D})
$$

instead of Eq. (2.9) with the nonlocal averaging process (2.3).

\subsection{Effective or "dynamic" distance - Vanishing in- ternal length}

In a plain and uncracked medium the internal length and internal time concepts are equivalent as

$$
\|\boldsymbol{x}-\boldsymbol{s}\|=c \tau_{x s}
$$


with $c$ the information celerity taken as the wave speed. Then, if the internal time is related to the internal length as $l_{c}=c \tau_{c}$ the weight functions are equal,

$$
\psi\left(\frac{\|\boldsymbol{x}-\boldsymbol{s}\|}{l_{c}}\right)=\psi\left(\frac{\tau_{x s}}{\tau_{c}}\right)
$$

In a homogeneously damaged medium at $D=D_{0}$, the wave speed is proportional to the square root of the damaged Young's modulus and depends on the damage level as

$$
\tilde{c}=c \sqrt{1-D_{0}}
$$

One has in this case

$$
\tau_{x s}=\frac{\|x-s\|}{\tilde{c}}
$$

and

$$
\frac{\tau_{x s}}{\tau_{c}}=\frac{\|\boldsymbol{x}-\boldsymbol{s}\|}{\tilde{c} \tau_{c}}=\frac{\|\boldsymbol{x}-\boldsymbol{s}\|}{l_{c} \sqrt{1-D_{0}}}>\frac{\|\boldsymbol{x}-\boldsymbol{s}\|}{l_{c}}
$$

which shows that the effective or "dynamic" distance

$$
\|x-s\|_{\mathrm{eff}}=\frac{\|\boldsymbol{x}-\boldsymbol{s}\|}{\sqrt{1-D_{0}}}
$$

between two points increases, as expected, with damage. Eq. (2.17) defines in an equivalent manner an effective internal length

$$
\tilde{l}_{c}=l_{c} \sqrt{1-D_{0}}
$$

which tends to zero when $D_{0}$ tends to unity in accordance with Pijaudier-Cabot et al. results [4] of a material behavior becoming local on free edges (at least in the direction normal to the edge).

In $1 \mathrm{D}$ and in the non uniform case, the effective distance is defined as the integral over the path $[s, x]$ as $\left|\int_{s}^{x}\left(1-D\left(x^{\prime}\right)\right)^{-1 / 2} d x^{\prime}\right|$, in $3 \mathrm{D}$ as $c \tau_{x s}$ 


\section{Chapter 3}

\section{Equivalence between a crack and a damaged zone}

In order to illustrate the formulation ability, consider the specimens of figure 3.1: (a) a square plate, (b) a notched specimen and (c) a specimen with a damaged zone at $D=D_{0}$. Vertical $z$-acceleration fields are also plotted at the same instant for the 3 specimens. It is clearly noticed that the information initiated as an impulse at $\boldsymbol{x}=\boldsymbol{x}_{1}$ takes longer to reach point $x_{6}$ for both notched and damaged specimens (the wave generated in the example has to turn around the notch). Note the waves reflexion on upper notch and on upper damaged zone sides. When the damage $D_{0}$ becomes large (0.99 in the figure), notched and damaged specimens are found equivalent.
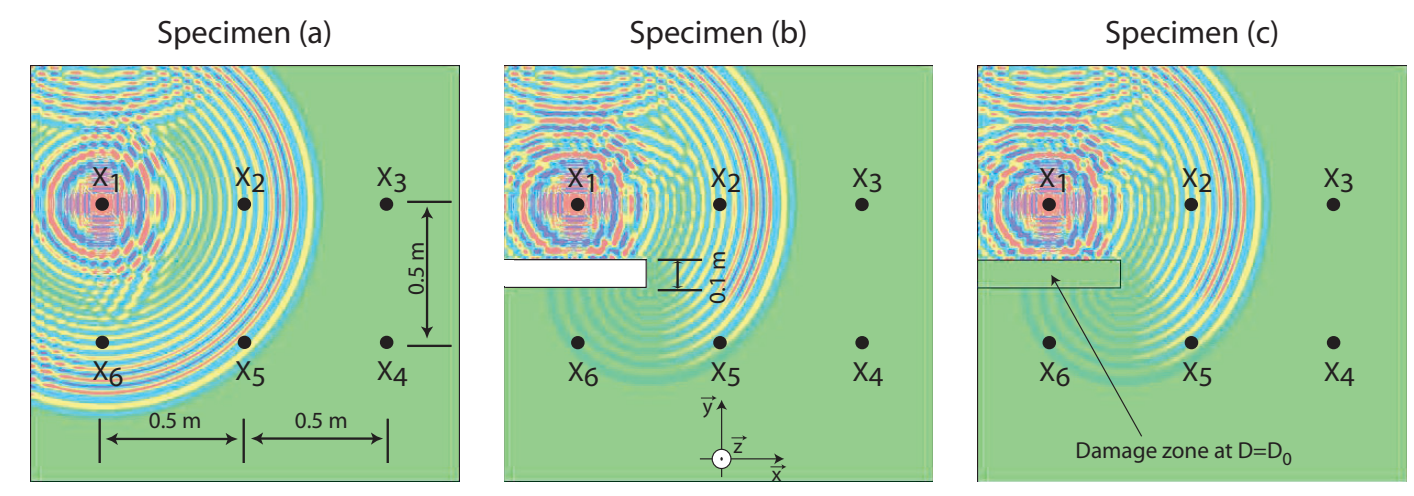

Figure 3.1: Square plate, notched and damaged specimens. Geometry and pictures of wave propagation at the same instant. 


\subsection{Connectivity matrices}

To quantify the approach, consider six points $\boldsymbol{x}_{p}$ in these specimens. The distances between these points, used in the classical nonlocal theory, are calculated in table 1 for the plate (a) (with $l=0.5 \mathrm{~m}$ ).

\begin{tabular}{c||c|c|c|c|c|c}
\hline \hline$\frac{\left\|\boldsymbol{x}_{p}-\boldsymbol{x}_{q}\right\|}{l_{c}}$ & $\boldsymbol{x}_{1}$ & $\boldsymbol{x}_{2}$ & $\boldsymbol{x}_{3}$ & $\boldsymbol{x}_{4}$ & $\boldsymbol{x}_{5}$ & $\boldsymbol{x}_{6}$ \\
\hline \hline $\boldsymbol{x}_{1}$ & 0 & $l / l_{c}$ & $2 l / l_{c}$ & $l \sqrt{5} / l_{c}$ & $l \sqrt{2} / l_{c}$ & $l / l_{c}$ \\
\hline $\boldsymbol{x}_{2}$ & $l / l_{c}$ & 0 & $l / l_{c}$ & $l \sqrt{2} / l_{c}$ & $l / l_{c}$ & $l \sqrt{2} / l_{c}$ \\
\hline$x_{3}$ & $2 l / l_{c}$ & $l / l_{c}$ & 0 & $l / l_{c}$ & $l \sqrt{2} / l_{c}$ & $l \sqrt{5} / l_{c}$ \\
\hline$x_{4}$ & $l \sqrt{5} / l_{c}$ & $l \sqrt{2} / l_{c}$ & $l / l_{c}$ & 0 & $l / l_{c}$ & $2 l / l_{c}$ \\
\hline$x_{5}$ & $l \sqrt{2} / l_{c}$ & $l / l_{c}$ & $l \sqrt{2} / l_{c}$ & $l / l_{c}$ & 0 & $l / l_{c}$ \\
\hline$x_{6}$ & $l / l_{c}$ & $l \sqrt{2} / l_{c}$ & $l \sqrt{5} / l_{c}$ & $2 l / l_{c}$ & $l / l_{c}$ & 0 \\
\hline
\end{tabular}

Table 3.1: Connectivity table built from the classical distances $\|\boldsymbol{x}-\boldsymbol{s}\|=\left\|\boldsymbol{x}_{p}-\boldsymbol{x}_{q}\right\|$ 
The connectivity table is rewritten in a matrix form as a connectivity matrix ( $\sqrt{2}$ and $\sqrt{5}$ replaced by their numerical values to simplify further comparisons),

$$
\boldsymbol{C}=\frac{l}{l_{c}} \cdot\left(\begin{array}{cccccc}
0 & 1 & 2 & 2.236 & 1.414 & 1 \\
1 & 0 & 1 & 1.414 & 1 & 1.414 \\
2 & 1 & 0 & 1 & 1.414 & 2.236 \\
2.236 & 1.414 & 1 & 0 & 1 & 2 \\
1.414 & 1 & 1.414 & 1 & 0 & 1 \\
1 & 1.414 & 2.236 & 2 & 1 & 0
\end{array}\right)
$$

The classical connectivity matrix $\boldsymbol{C}$ is the same (and is symmetric) for the 3 structures (a), (b) and (c). It does not take into account the presence of notches nor the occurence of damage as $C_{16}=C_{25}=C_{34}=l / l_{c}$.

In order to determine the connectivity matrix with the new internal time formulation, one proceeds as follows:

- an impulse force according to the out of plane $z$-axis (close to a Dirac) is applied at each point $\boldsymbol{x}=\boldsymbol{x}_{p}$ in a dynamic finite element analysis of a 3D model of a plate (thickness of $0.1 \mathrm{~m}, 720000$ nodes and 360000 elements, free boundary conditions),

- the times $\tau_{x s}$ at which the first pic of the $z$-acceleration information arrives at point $s=x_{q}$ are recorded. Divided by $\tau_{c}$ they are put in the form of connectivity matrices $C_{\tau}$,

- the expressions for $\tau_{x s} / \tau_{c}$ are synthesized by use of the relation $l_{c}=c \tau_{c}$ in the form $\boldsymbol{C}_{\tau}=\frac{l}{l_{c}} \cdot \boldsymbol{A}$ and compared to table 1 results for the different specimens.

For the concrete square plate and notched specimen one ends up to $(E=35000 \mathrm{MPa}$, $\left.\nu=0.2, \rho=2400 \mathrm{~kg} / \mathrm{m}^{3}\right)$ :

$$
\begin{gathered}
\boldsymbol{C}_{\tau}^{(a)}=\frac{l}{l_{c}} \cdot\left(\begin{array}{cccccc}
0 & 1 & 2.008 & 2.233 & 1.420 & 1 \\
1 & 0 & 1 & 1.420 & 1 & 1.420 \\
2.008 & 1 & 0 & 1 & 1.420 & 2.233 \\
2.233 & 1.420 & 1 & 0 & 1 & 2.008 \\
1.420 & 1 & 1.420 & 1 & 0 & 1 \\
1 & 1.420 & 2.233 & 2.008 & 1 & 0
\end{array}\right) \\
\boldsymbol{C}_{\tau}^{(b)}=\frac{l}{l_{c}} \cdot\left(\begin{array}{cccccc}
0 & 1 & 2.008 & 2.233 & 1.420 & 1.494 \\
1 & 0 & 1 & 1.420 & 1 & 1.420 \\
2.008 & 1 & 0 & 1 & 1.420 & 2.233 \\
2.233 & 1.420 & 1 & 0 & 1 & 2.008 \\
1.420 & 1 & 1.420 & 1 & 0 & 1 \\
1.494 & 1.420 & 2.233 & 2.008 & 1 & 0
\end{array}\right)
\end{gathered}
$$


where the notch presence is taken into account (boxed terms), leading for the notched specimen to $C_{\tau 16}^{(b)}=\tau_{16}^{(b)} / \tau_{c}=1.494 l / l_{c}$ intead of $C_{\tau 16}^{(a)}=\tau_{16}^{(a)} / \tau_{c}=l / l_{c}$ for the plate with no notch.

For the specimen with the damaged zone at $D=D_{0}=0.99$, the connectivity matrix corresponding to the nonlocal internal time analysis reads

$$
\boldsymbol{C}_{\tau}^{(c)}=\frac{l}{l_{c}} \cdot\left(\begin{array}{cccccc}
0 & 1 & 2.008 & 2.233 & 1.420 & 1.500 \\
1 & 0 & 1 & 1.420 & 1 & 1.420 \\
2.008 & 1 & 0 & 1 & 1.420 & 2.233 \\
2.233 & 1.420 & 1 & 0 & 1 & 2.008 \\
1.420 & 1 & 1.420 & 1 & 0 & 1 \\
1.500 & 1.420 & 2.233 & 2.008 & 1 & 0
\end{array}\right)
$$

Again ony the terms 16 and 61 of the connectivity matrices are changed and found close to the value $1.494 l / l_{c}$ obtained with reals notches: the damaged zone behaves as a notch, damage and notch being both taken into account by the proposed nonlocal with internal time analysis.

\subsection{Straight 1D wave propagation}

For a better understanding, focus on times $\tau_{16}, \tau_{25}, \tau_{34}$ wave propagation in specimen (c) from $\boldsymbol{x}_{1}$ to $\boldsymbol{x}_{6}$, from $\boldsymbol{x}_{2}$ to $\boldsymbol{x}_{5}$, from $\boldsymbol{x}_{3}$ to $\boldsymbol{x}_{4}$. The corresponding distances are equal in the 3 cases (equal to $l$ ). Due to the presence of the notch the time $\tau_{16}$ is of course larger than the time $\tau_{25}$ correspnding to a straight path with no notch (and $\tau_{25} / \tau_{c}=l / l_{c}$ ).

A wave propagating along the path $\boldsymbol{x}_{1}-\boldsymbol{x}_{6}$ in specimen (c) slows down to the celerity $\tilde{c}$ when meeting the damaged zone. If one only consider the straight path $\boldsymbol{x}_{1}-\boldsymbol{x}_{6}$ for the wave propagation, one has

$$
\tau_{16}=\frac{l}{c}+\frac{e}{c} \cdot \frac{1-\sqrt{1-D_{0}}}{\sqrt{1-D_{0}}} \quad \text { or } \quad \frac{\tau_{16}}{\tau_{c}}=\frac{l}{l_{c}}\left[1+\frac{e}{l} \cdot \frac{1-\sqrt{1-D_{0}}}{\sqrt{1-D_{0}}}\right]
$$

where $e$ is the thickness of the damaged zone at $D=D_{0}$.

The figure 3.2 shows the normalized increase of time $\tau_{34}=\tau_{34}^{(c)}$ with respect to the damage value $D_{0}$ and for different ratios $e / l$. The ratio $\left(\tau_{34} / \tau_{c}\right) /\left(l / l_{c}\right)$ in case (c) is equal (resp. close) to unity for a zero (resp. small) damage, the nonlocal theory with internal time recovering then the classical nonlocal theory. The very large increase obtained for large values of the damage, enhanced by a large damaged zone thickness, proves that both a real crack and a highly damaged zone are equivalent in the proposed nonlocal framework. This property is emphasized when the ratio of the weight functions $\psi\left(\tau_{34} / \tau_{c}\right) / \psi\left(l / l_{c}\right)$ is 
drawn (figures 3.3 to fig:ratioexp02) for $l / l c=5,1$ and 0.2 and for the Gaussian weight function $\psi(\xi)=e^{-\frac{1}{2} \xi^{2}}$ : the nonlocal spatial interaction between points across a damaged zone strongly diminishes with damage increase, the larger the points distance $l$ with respect to the internal length $l_{c}$, the stronger the effect.

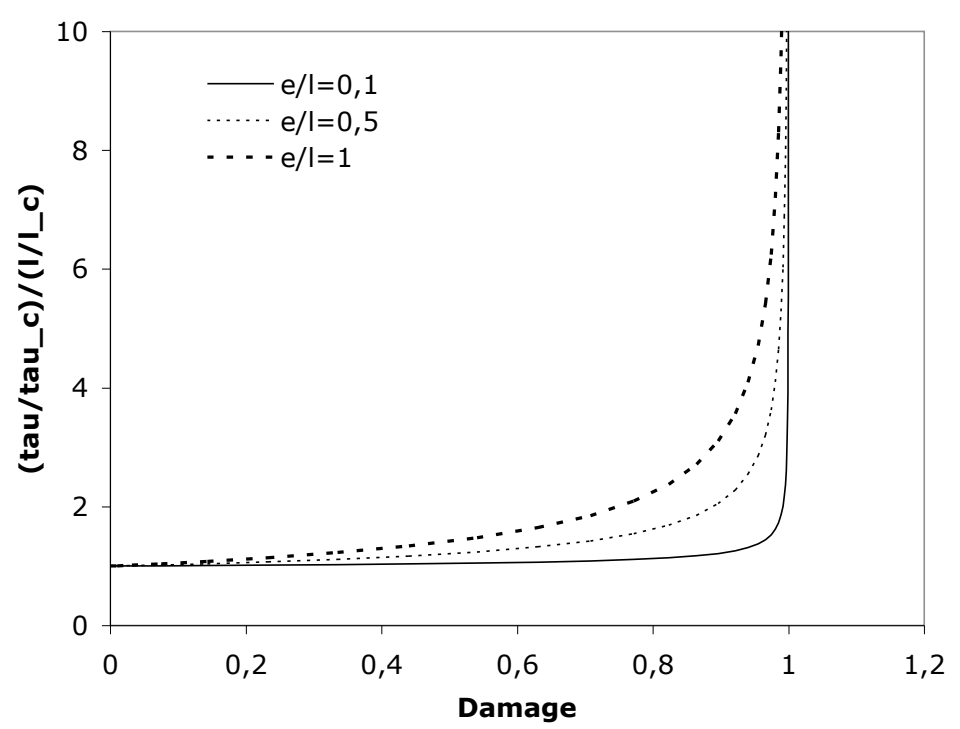

Figure 3.2: Ratio $\frac{\tau_{34}}{\tau_{c}} / \frac{l}{l_{c}}$ versus damage $D_{0}$. 


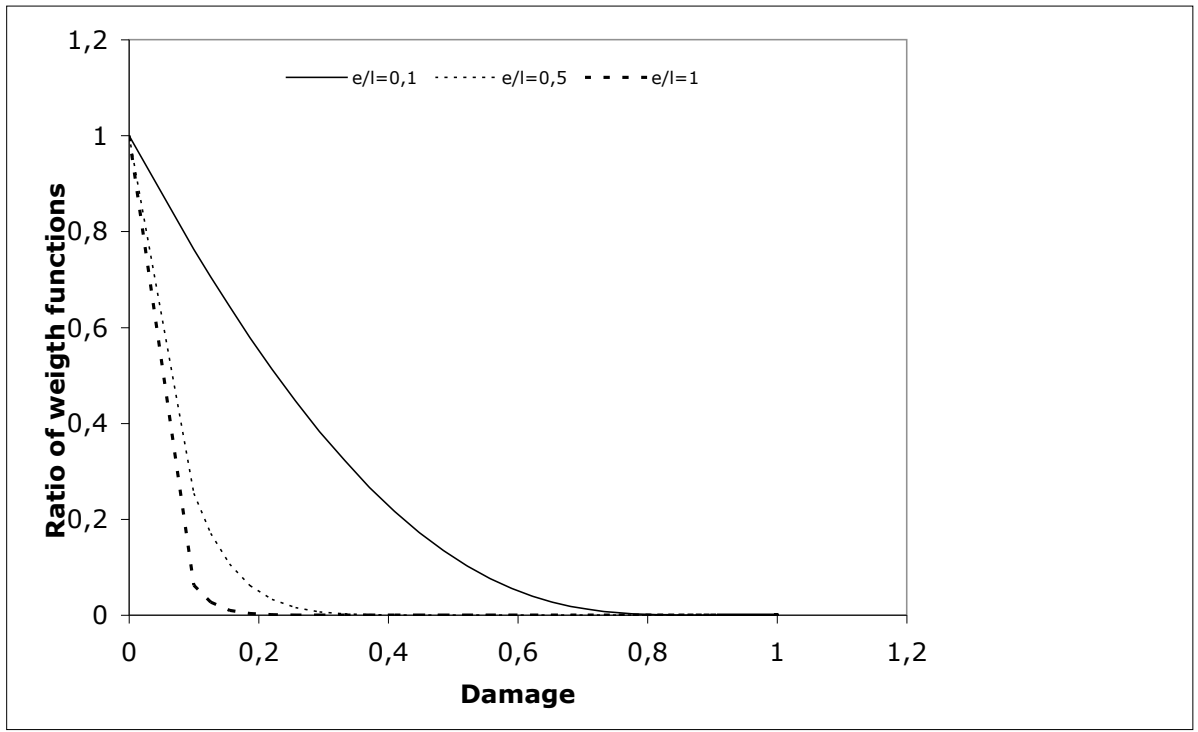

Figure 3.3: Ratio $\frac{\exp \left(-\frac{\tau_{34}^{2}}{\tau_{c}^{2}}\right)}{\exp \left(-\frac{l^{2}}{l_{c}^{2}}\right)}$ versus damage $D_{0}$ for $l / l_{c}=5$. 


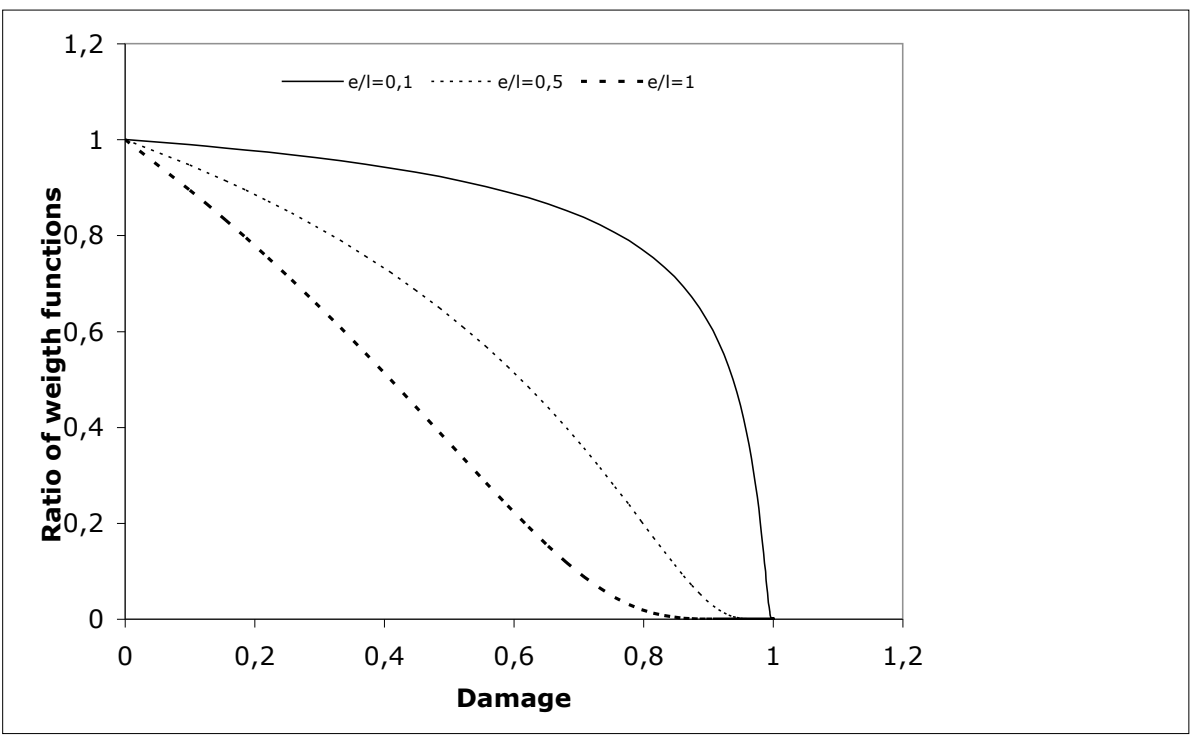

Figure 3.4: Ratio $\frac{\exp \left(-\frac{\tau_{34}^{2}}{\tau_{c}^{2}}\right)}{\exp \left(-\frac{l^{2}}{l_{c}^{2}}\right)}$ versus damage $D_{0}$ for $l / l_{c}=1$. 


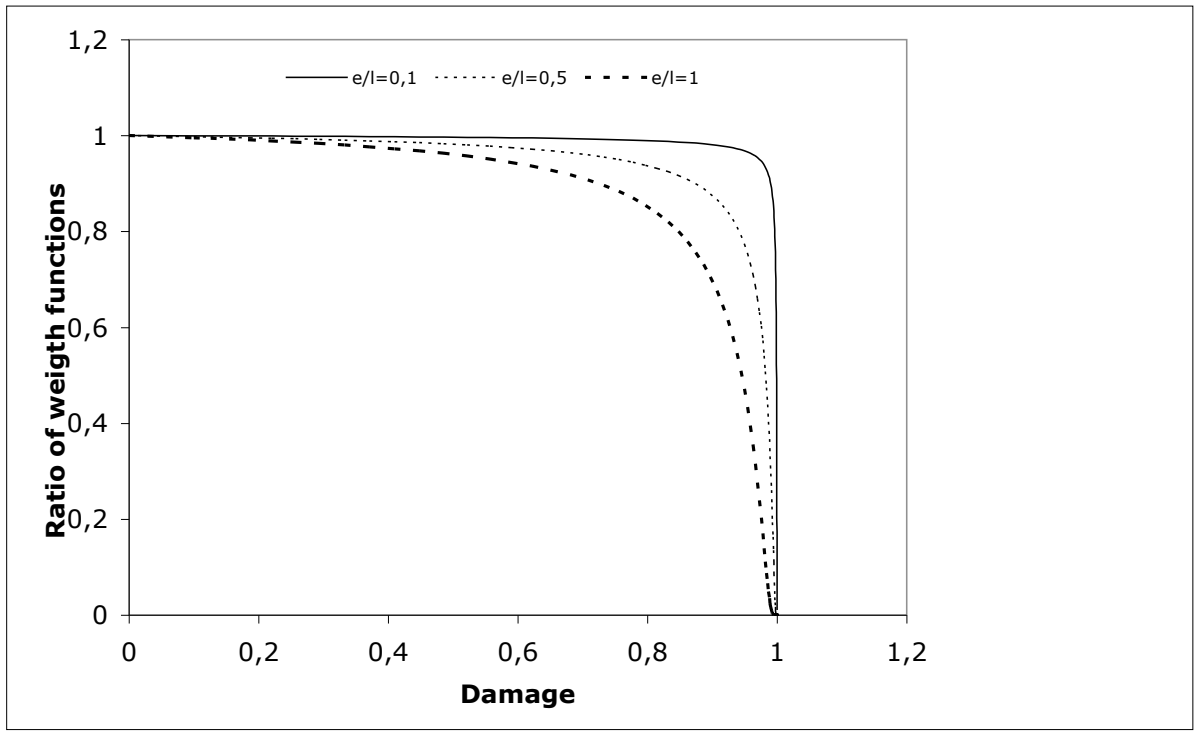

Figure 3.5: Ratio $\frac{\exp \left(-\frac{\tau_{34}^{2}}{\tau_{c}^{2}}\right)}{\exp \left(-\frac{l^{2}}{l_{c}^{2}}\right)}$ versus damage $D_{0}$ for $l / l_{c}=0.2$. 


\section{Chapter 4}

\section{Bar with a damaged zone}

As an example emphasizing how a damaged zone is taken into account, consider a bar in tension at a stress level $\sigma$. The length of the bar is $2 L$, a damaged zone at large $D=D_{0}$ corresponds to $x \in[-l, l]$. The non local analyses perform the integrals (2.1) over the whole bar,

$$
\epsilon^{n l}(x)=\frac{1}{V_{r}} \int_{-L}^{L} \psi \cdot \epsilon(s) d s \quad V_{r}(x)=\int_{-L}^{L} \psi d s
$$

with as strain $\epsilon(x)=\sigma / E$ if $|x|>l, \epsilon(x)=\sigma / E\left(1-D_{0}\right)$ else and where $\psi=\psi\left(\frac{|x-s|}{l_{c}}\right)$ for the classical nonlocal analysis, $\psi=\psi\left(\frac{\tau_{x s}}{\tau_{c}}\right)$ for the nonlocal analysis with internal time. The Gaussian weight function $\psi(\xi)=e^{-\frac{1}{2} \xi^{2}}$ is considered. The normalizing factors $V_{r}(x)$ are compared for both analyses in figure 4.1a where $D_{0}=0.99, l=2.5 \mathrm{~cm}, L=50 \mathrm{~cm}$ and where the characteristic length is taken as $l_{c}=10 \mathrm{~cm}$ (twice the size $2 l$ of the damaged zone). The classical normalization does not "see" the damaged zone and averages across it when the new nonlocal with internal time approach behaves for the undamaged domains almost as for two independent bars, as expected. The nonlocal strains obtained with both approaches are compared in figure 4.1b. In this particular piecewise constant strain field, the formulation with internal time gives as expected a nonlocal strain field closer to the local strain field than the classical nonlocal approach for which too much importance is given to points over the damaged zone when performing the integral (4.1). Note that in case of structural failure, strain localization leads to non homogeneous fields. The proposed nonlocal averaging then fully acts and makes the solution regular. 

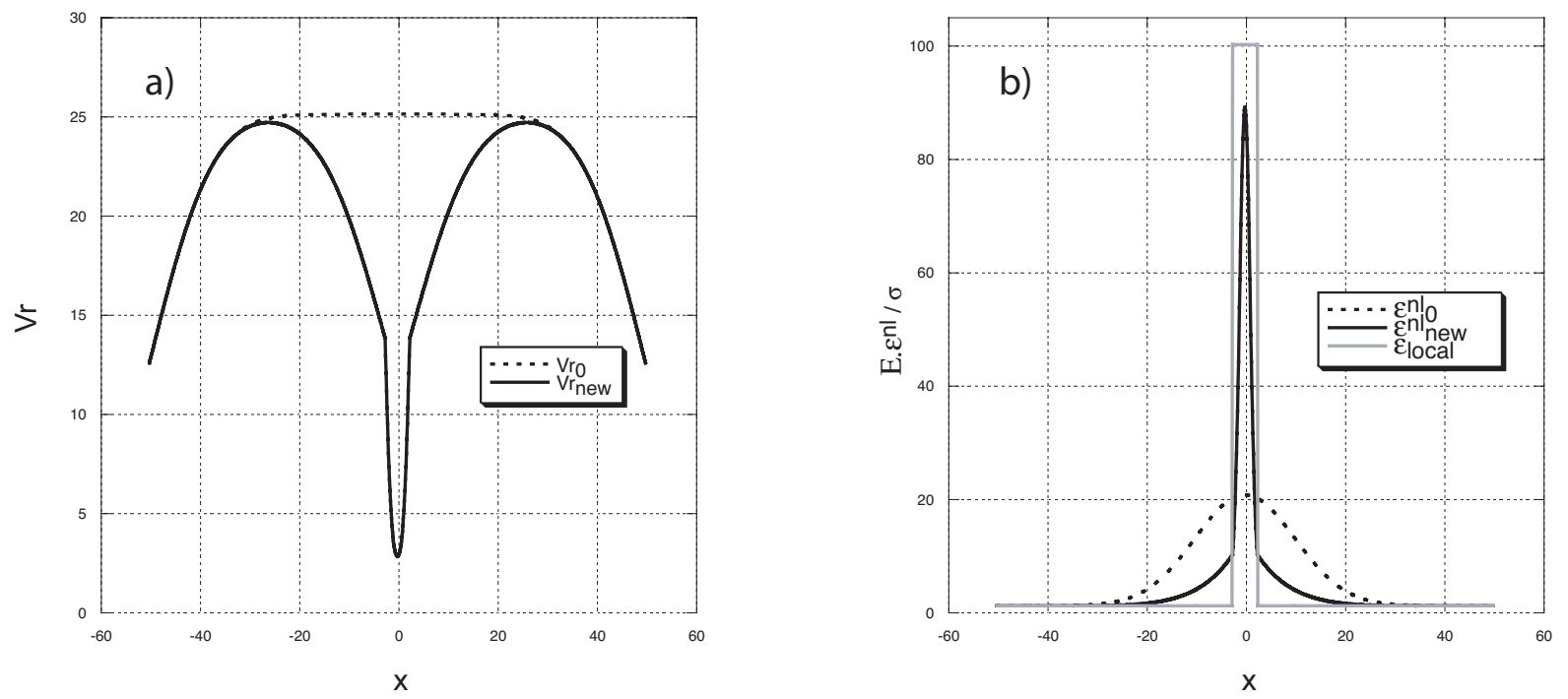

Figure 4.1: a) Comparison of normalizing factors $V_{r}(x)$; b) Comparison of normalized nonlocal strains (lengths in cm, black: nonlocal with internal time, grey: classical nonlocal, dot: local strain). 


\section{Conclusion}

A new nonlocal integral formulation is proposed. An internal time is introduced leading to the equivalent definition of an effective or "dynamic" distance and of an evolving internal length. The important feature is that the distance bewteen points is not the argument of the averaging weight function anymore. It is replaced by the information or wave time propagation between these points. The nonlocal weight function build is then symmetric, even in non homogeneous bodies. Pre-computations of wave propagation in the considered structure allow to build the corresponding nonlocal connectivity matrix, with of course the open question of the wave type.

The cracks and notches presence - and if necessary their closure - are naturally taken into account within the wave propagation study and Pijaudier-Cabot and Bazant nonlocal theory is recovered far from the boundaries. The proposed approach makes equivalent a crack and a highly damaged zone, as points across a notch have a small contribution in the nonlocal averaging.

Important point, no assumption on the medium isotropy is made. The proposed nonlocal framework includes anisotropy, either initial or induced.

Last, when no damage is considered as in plasticity with negative hardening models, the internal time concept may still be used, for example by making nonlocal - through Eq. (2.3) - the accumulated plastic strain. The presence of existing notches and cracks will be naturally taken into account if propagation of elastic waves defines the time $\tau_{x s}$ but the equivalence between a localized zone and a crack will not be obtained. To gain this last feature, two possibilities are:

- to consider an elasticity coupled with damage even if the plasticity part of the model remain not affected by damage,

- to define $\tau_{x s}$ from plastic waves propagation (no need of damage then). 


\section{Bibliography}

[1] Pijaudier-Cabot G., Bazant Z., J. Engng Mech., ASCE, 113, pp. 1512-33, 1987.

[2] Aifantis E. Int J Plast, 3, pp. 211-47, 1987.

[3] Peerlings R., de Borst R., Brekelmans W., de Vree J., Int. J. Numer. Methods Engng, 39, pp. 391-403, 1996.

[4] Pijaudier-Cabot G., Krayani A., Dufour F., chapter in Nonlocal Modeling of Materials Failure, H. Yuan and F.H. Wittmann Ed., Aedificio Pubs, 2007.

[5] Geers M., de Borst R., Brekelmans W., Peerlings R., Comput. Methods. Appl. Mech. Engng, 160, 133-153, 1998.

[6] Pijaudier-Cabot G., Haidar K., Dubé J.-F., Int. J. Num. Anal. Meth. Geomech., 28, pp. 633-652, 2004.

[7] Needleman A., Comp. Meth. Mech. Engng, 67, 69-85, 1988.

[8] Denoual C. and Hild F., Comp. Meth. Appl. Mech. Eng., 183 (3-4), pp. 247-258, 2000.

[9] Allix O. and Deü J.F., Engineering Transactions, 45, pp. 29-46, 1997.

[10] Jirasek M., Int. J. Engng Science, 4, pp. 1553-1602, 2003.

[11] Mazars J., and Berthaud Y., and Ramtani, S., The unilateral behavior of damage concrete, Eng. Fract. Mech., 35, pp. 629-635, 1990.

[12] Desmorat R., Modèle d'endommagement anisotrope avec forte dissymétrie traction/compression, 5è journéees du Regroupement Francophone pour la Recherche et la Formation sur le Béton (RF2B), Liège, Belgium, 5-6 july, 2004.

[13] Desmorat R., Gatuingt F, Ragueneau F., Nonlocal anisotropic damage model and related computational aspects for quasi-brittle materials, Engineering Fracture mechanics, 74, p. 1539-1560, 2007. 